\title{
Artificial Intelligence to Predict Mortality in Critically ill COVID-19 Patients Using Data from the First 24h: A Case Study from Lombardy Outbreak
}

\section{Massimiliano Greco}

HUNIMED: Humanitas University

Giovanni Angelotti

IRCCS Humanitas Research Hospital

Pier Francesco Caruso ( $\nabla$ pierfrancesco.caruso@humanitas.it)

HUNIMED: Humanitas University https://orcid.org/0000-0002-0839-4396

Alberto Zanella

Fondazione IRCCS Ca' Granda Ospedale Maggiore Policlinico

Niccolò Stomeo

IRCCS Humanitas Research Hospital

\section{Elena Costantini}

IRCCS Humanitas Research Hospital

\section{Alessandro Protti}

HUNIMED: Humanitas University

\section{Antonio Pesenti}

Fondazione IRCCS Ca' Granda Ospedale Maggiore Policlinico

\section{Giacomo Grasselli}

Fondazione IRCCS Ca' Granda Ospedale Maggiore Policlinico

\section{Maurizio Cecconi}

HUNIMED: Humanitas University

\section{Research}

Keywords: COVID-19, machine learning, ICU management, emergency organization, outcomes, epidemiology

Posted Date: May 24th, 2021

DOI: https://doi.org/10.21203/rs.3.rs-508325/v1

License: (c) (i) This work is licensed under a Creative Commons Attribution 4.0 International License. Read Full License 


\section{Abstract}

Introduction: SARS-CoV-2 infection was first identified at the end of 2019 in China, and subsequently spread globally. COVID-19 disease frequently affects the lungs leading to bilateral viral pneumonia, progressing in some cases to severe respiratory failure requiring ICU admission and mechanical ventilation. Risk stratification at ICU admission is fundamental for resource allocation and decision making, considering that baseline comorbidities, age, and patient conditions at admission have been associated to poorer outcomes. Supervised machine learning techniques are increasingly diffuse in clinical medicine and can predict mortality and test associations reaching high predictive performance. We assessed performances of a machine learning approach to predict mortality in COVID-19 patients admitted to ICU using data from the Lombardy ICU Network.

Methods: this is a secondary analysis of prospectively collected data from Lombardy ICU network. To predict survival at 7-,14- and 28 days we built two different models; model $\mathrm{A}$ included patient demographics, medications before admission and comorbidities, while model B also included the data of the first day since ICU admission. 10-fold cross validation was repeated 2500 times, to ensure optimal hyperparameter choice. The only constrain imposed to model optimization was the choice of logistic regression as final layer to increase clinical interpretability. Different imputation and over-sampling techniques were employed in model training.

Results 1503 patients were included, with 766 deaths (51\%). Exploratory analysis and Kaplan-Meier curves demonstrated mortality association with age and gender. Model $A$ and $B$ reached the greatest predictive performance at 28 days (AUC 0.77 and 0.79), with lower performance at 14 days (AUC 0.72 and 0.74 ) and 7 days (AUC 0.68 and 0.71 ). Male gender, age and number of comorbidities were strongly associated with mortality in both models. Among comorbidities, chronic kidney disease and chronic obstructive pulmonary disease demonstrated association. Mode of ventilatory assistance at ICU admission and Fraction of Inspired oxygen were associated with mortality in model B.

Conclusions Supervised machine learning models demonstrated good performance in prediction of 28day mortality. 7-days and 14-days predictions demonstrated lower performance. Machine learning techniques may be useful in emergency phases to reach higher predictive performance with reduced human supervision using complex data.

\section{Introduction}

Towards the end of 2019, a novel strand of coronavirus, named Severe Acute Respiratory Syndrome coronavirus-2 (SARS-CoV-2) was identified as the causative agent of an outbreak of bilateral pneumonia in the city of Wuhan in China.(1) The clinical picture related with SARS-CoV-2 infection, was subsequently named COVID-19 disease, and is frequently characterized by severe bilateral pneumonia. The epidemic spread outside mainland China to an increasing number of countries, and on March 11th, 2020 it was declared a pandemic.(2) 
Italy, and in particular Lombardy, was the epicenter of the first outbreak of COVID-19 in the Western World. In Lombardy, the first cases were recognized at the end of February, and the number of Intensive Care Unit admissions rose substantially in the following weeks.(3)

The outcomes of patients admitted to ICU for COVID-19 disease are severe, and comparable with those of patients with severe Acute Respiratory Distress Syndrome (ARDS), with mortality around $50 \%$ in patients requiring mechanical ventilation. (4-6). Several factors have been associated with a negative outcome, including age, male gender, previous comorbidities, and level of respiratory support at ICU admission (4, 7). Machine learning algorithms are increasingly employed in clinical medicine due to their potential of analysing large amount of information with reduced human supervision, resulting in high predictive performance. $(8,9)$ These models can similarly help to hasten data cleaning and finetuning of predictive models, a process which would draw large resources during an emergency. Increased predictive performance using artificial intelligence could be useful both for patients, to target the best therapeutic strategy with realistic goals, and for the healthcare system to enhance the allocation of resources. To assess the performance of a machine learning approach on operative data collected during the upsurge phase of the pandemic we propose a supervised learning model to predict mortality in COVID-19 critically ill patients.

\section{Methods}

This is a secondary analysis of data collected during the COVID-19 Lombardy outbreak from February 2020 to April 2020 using operational and clinical data from the Lombardy ICU network, as described in previous studies. $(3,4,10)$

The aim of this study is to predict survival at 7, 14 and 28 days from ICU admission, using a typical supervised learning framework.

Data on patient baseline characteristics, including medications, comorbidities and baseline ventilation parameters are included in the analysis. Data are described as mean (standard deviation) or frequency (percentage), as appropriate.

We conducted an initial exploratory analysis of data, testing univariate and bivariate associations with Chi-square test and Mann-Whitney $U$ test. Survival analysis was conducted plotting Kaplan-Meier curves. We built two different models ( $A$ and $B$ ), to predict survival at three different timepoints: 7-day, 14-day and 28-day mortality from ICU admission. Model A included only baseline patient data (age, gender, home medications and comorbidities); Model B included baseline data and data from the first $24 \mathrm{~h}$ in ICU. The only imposition to models was on the last layer: data output had to be expressed through a logistic regression so that interpretability was not lost and we were able to better understand the models' decision-making process.

Both models were trained using 10 -fold cross validation. Hyperparameters were optimized to maximize the out-of-fold area under the curve (AUC) on a randomized grid space. Cross- validation was repeated 
2500 times to ensure optimal hyperparameter choice, for a grand total of 25.000 effectively trained models. To avoid selection bias during modeling, hyperparameters optimization was conducted with a high degree of freedom, with the only constraint of using a logistic regression as final layer of the model, to retain some native interpretability. During training, the optimization process had the choice of using different over-sampling techniques (Synthetic Minority Over-sampling Technique [SMOTE], support-vector machine- Synthetic Minority Over-sampling Technique [SVM-SMOTE] or Adaptive synthetic sampling approach for imbalanced learning [ADASYN]),(11-13) as well as different imputation techniques.

We opted to validate the model using cross-validations compared to hold-out validations to account for the limited observation space.

\section{Results}

We included a total of 1503 patients in the analysis. 28-days mortality was $51 \%(n=766)$. Survivors were significantly younger and suffered fewer comorbidities at admission compared to non-survivors $(\mathrm{p}<$ 0.05). $44 \%$ of survivors had no pre-existing comorbidities, compared to $26 \%$ of non-survivors (Table 1 ).

Table 1

Description of baseline characteristics according to survival. Q1-Q3 are thresholds for interquartile range.

\begin{tabular}{|c|c|c|c|c|c|c|c|c|c|c|}
\hline \multirow[t]{2}{*}{ Outcome } & \multirow[t]{2}{*}{$\begin{array}{l}\text { Patients } \\
\text { (n) }\end{array}$} & \multirow[t]{2}{*}{$\begin{array}{l}\text { Male } \\
\text { [\%] }\end{array}$} & \multicolumn{2}{|c|}{$\begin{array}{l}\text { ICU stay } \\
\text { (days) }\end{array}$} & \multicolumn{4}{|c|}{ Age (years) } & \multirow{2}{*}{$\begin{array}{l}\text { Mean number } \\
\text { of } \\
\text { comorbidities } \\
\text { (SD) }\end{array}$} & \multirow[t]{2}{*}{$\begin{array}{l}\text { No previous } \\
\text { comorbidities }\end{array}$} \\
\hline & & & q1 & q2 & q3 & $\mathrm{q} 1$ & q2 & q3 & & \\
\hline Alive & 735 & $78.10 \%$ & 7 & 12 & 19 & 52 & 59 & 65 & $1.05(1.23)$ & $44.25 \%$ \\
\hline Death & 768 & $84.90 \%$ & 5 & 10 & 15 & 62 & 68 & 73 & $1.75(1.50)$ & $25.73 \%$ \\
\hline Tot & 1503 & $81.60 \%$ & 6 & 11 & 17 & 56 & 64 & 70 & $1.40(1.42)$ & $34.86 \%$ \\
\hline
\end{tabular}


Table 2

Cross-validation performance scores for model A and B.

\begin{tabular}{|c|c|c|c|c|c|c|c|c|}
\hline \multirow[t]{2}{*}{ Model A } & \multicolumn{2}{|l|}{ AUC } & \multicolumn{2}{|c|}{ Precision } & \multicolumn{2}{|l|}{ Recall } & \multicolumn{2}{|l|}{$\mathbf{F} 1$} \\
\hline & Mean & SD & Mean & SD & Mean & SD & Mean & SD \\
\hline y7 & 0.68 & 0.09 & 0.58 & 0.04 & 0.64 & 0.08 & 0.54 & 0.06 \\
\hline y14 & 0.72 & 0.04 & 0.64 & 0.04 & 0.65 & 0.04 & 0.63 & 0.04 \\
\hline y28 & 0.77 & 0.04 & 0.7 & 0.05 & 0.7 & 0.05 & 0.7 & 0.05 \\
\hline \multirow[t]{2}{*}{ Model B } & \multicolumn{2}{|l|}{ AUC } & \multicolumn{2}{|c|}{ Precision } & \multicolumn{2}{|l|}{ Recall } & \multicolumn{2}{|l|}{$\mathbf{F} 1$} \\
\hline & Mean & SD & Mean & SD & Mean & SD & Mean & SD \\
\hline y7 & 0.71 & 0.08 & 0.59 & 0.03 & 0.66 & 0.07 & 0.57 & 0.04 \\
\hline y14 & 0.74 & 0.04 & 0.67 & 0.03 & 0.68 & 0.03 & 0.66 & 0.04 \\
\hline y28 & 0.79 & 0.04 & 0.72 & 0.04 & 0.71 & 0.04 & 0.71 & 0.04 \\
\hline
\end{tabular}

Table 3

Model A and B performance predicting 28-days survival

\begin{tabular}{|lllllll|}
\hline & \multicolumn{5}{c}{ Survivors } & \multicolumn{4}{l|}{ Non survivors } \\
\cline { 2 - 7 } & F1 & Precision & Recall & F1 & Precision & Recall \\
& mean (SD) & mean (SD) & mean (SD) & mean (SD) & mean (SD) & mean (SD) \\
\hline Model A & $0.71(0.05)$ & $0.69(0.05)$ & $0.72(0.06)$ & $0.69(0.06)$ & $0.71(0.05)$ & $0.68(0.07)$ \\
\hline Model B & $0.72(0.04)$ & $0.71(0.05)$ & $0.73(0.04)$ & $0.71(0.05)$ & $0.72(0.04)$ & $0.70(0.06)$ \\
\hline $\begin{array}{l}\text { Precision is also known as Positive Predictive Value, recall also known as sensitivity. F1 combines } \\
\text { precision and recall. SD = Standard Deviation }\end{array}$
\end{tabular}


Table 4

Feature and relative odds from model A and B, for prediction of 28-days survival.

\begin{tabular}{|c|c|c|c|c|c|}
\hline Features Model A & odds & SD & Model B & odds & SD \\
\hline Liver disease & 1.363 & 0.076 & $\begin{array}{l}\text { Spontaneous ventilation } \\
\text { Admssion }\end{array}$ & 2.527 & 0.878 \\
\hline Other diseases & 1.295 & 0.045 & CPAP at admission & 1.872 & 0.173 \\
\hline Anti-hypertensive drugs & 1.238 & 0.045 & Liver disease & 1.434 & 0.189 \\
\hline Previous malignancy & 1.059 & 0.054 & Anti-hypertensive drugs & 1.327 & 0.062 \\
\hline Beta- blockers & 1.036 & 0.057 & $\begin{array}{l}\mathrm{PaO} 2 / \mathrm{FiO} 2 \text { ratio at } \\
\text { admission }\end{array}$ & 1.305 & 0.109 \\
\hline Hypercholesterolemia & 1.014 & 0.074 & Other & 1.197 & 0.102 \\
\hline Heart disease & 0.987 & 0.037 & NIV at admission & 1.142 & 0.169 \\
\hline No previous comorbidity & 0.978 & 0.040 & $\mathrm{PaO} 2$ at admission & 1.040 & 0.047 \\
\hline Statin & 0.891 & 0.056 & Previous malignancy & 1.008 & 0.019 \\
\hline ARB & 0.886 & 0.042 & None & 0.998 & 0.020 \\
\hline Diuretics & 0.832 & 0.048 & Heart disease & 0.996 & 0.015 \\
\hline Immunosuppressant & 0.823 & 0.042 & Beta-blockers & 0.962 & 0.054 \\
\hline Diabetes mellitus & 0.815 & 0.025 & Hypercholesterolemia & 0.946 & 0.073 \\
\hline $\begin{array}{l}\text { Number of previous } \\
\text { comorbidities }\end{array}$ & 0.753 & 0.022 & Statin & 0.943 & 0.061 \\
\hline COPD & 0.737 & 0.031 & IMV at admission & 0.893 & 0.027 \\
\hline Gender & 0.698 & 0.032 & ARB & 0.8809 & 0.064 \\
\hline ACEI & 0.640 & 0.024 & Immunosuppressant_tot & 0.857 & 0.066 \\
\hline CKD & 0.580 & 0.021 & $\begin{array}{l}\text { Number of previous } \\
\text { comorbidities }\end{array}$ & 0.820 & 0.079 \\
\hline \multirow[t]{4}{*}{ Age } & 0.296 & 0.015 & Diabetes mellitus & 0.818 & 0.044 \\
\hline & & & PEEP level at admission & 0.813 & 0.030 \\
\hline & & & diuretics & 0.799800 & 0.084 \\
\hline & & & Gender & 0.663 & 0.036 \\
\hline
\end{tabular}

Angiotensin receptor blockers (ARB), ACE-I ace-inhibitors, CKD: chronic kidney disease, COPD : chronic obstructive pulmonary disease, $\mathrm{CPAP}=$ continuous positive airway pressure, NIV = Non invasive ventilation, IMV = Invasive ventilation, PEEP = Positive End-Expiratory Pressure, SD = Standard Deviation 


\begin{tabular}{|c|c|c|c|c|}
\hline Features Model A & odds & Model B & odds & SD \\
\hline & & ACE-I & 0.607 & 0.027 \\
\hline & & COPD & 0.603 & 0.068 \\
\hline & & FiO2 & 0.496 & 0.027 \\
\hline & & CKD & 0.2890 & 0.026 \\
\hline & & Age & 0.260 & 0.015 \\
\hline \multicolumn{5}{|c|}{$\begin{array}{l}\text { Angiotensin receptor blockers (ARB), ACE-I ace-inhibitors, CKD: chronic kidney disease, COPD : chronic } \\
\text { obstructive pulmonary disease, CPAP = continuous positive airway pressure, NIV = Non invasive } \\
\text { ventilation, IMV = Invasive ventilation, PEEP = Positive End-Expiratory Pressure, SD = Standard } \\
\text { Deviation }\end{array}$} \\
\hline
\end{tabular}

Male gender was associated with increased mortality $(p<0.05)$. Home medications and type of comorbidity also differed between survivors and non-survivors $(p<0.05)$, as reported in Fig. 1 , with a higher proportion of patients affected by liver disease among survivors.

Kaplan-Meier curves reveal a progressive reduction in survival from $70 \%$ at 10 days to $55 \%$ at 20 days from ICU admission reaching a plateau thereafter in both male and female populations (Supplemental Fig. 1and 2). Age was strongly associated with mortality, with 7-day survival ranging from $64 \%$ in the oldest age group to $93 \%$ in the 30-40 years group, with differences progressively increasing at 14 and 28days survival (Fig. 2).

Models A and B progressively increased their predictive performance with outcomes occurring later in time. Predicting survival at 7-days had intermediate performance in both model A and B - AUC of 0.68 and 0.71 for model A and B, respectively (Fig. 3).

The performance was similar at 14 days, with average AUCs 0.72 (model A) and 0.74 (model B).

The greatest predictive performance was reached at 28-days, with $A U C=0.77$ for model $A$ and $A U C=0.79$ for model B (Table II). Inter-class performance at 28-days displayed good-balance among all scores for both classes, with model A and B demonstrating similar precision and recall (0.7), and similar F1 scores (Table III).

Table IV reports mean odds for 28-days survival from model A and B. Feature importance seems rather coherent amongst the two models, with chronic kidney disease and age strongly associated with mortality.

\section{Discussion}

In this study we build two different models to predict survival in critically ill patients with COVID-19 disease, using supervised machine learning techniques. Model A included baseline characteristics only, while model B included baseline characteristics and ICU admission data. 
We assessed survival at 7-, 14- and 28- days for both models, reaching the highest predictive performance with later outcomes, an effect probably related to the increased balance between classes with increasing ICU stay.

Model B performance was reduced at 7 and 14-days. As model A included only baseline data and model $B$ included ventilatory parameters and other data at ICU admission, the loss in the predictive performance of model B can be attributed to a mixed effect (patients with worse respiratory but reduced baseline comorbidities may recover and stabilize, while older and more comorbid patients can still be at risk of deterioration after ICU admission). More complex and deeper models should probably be employed to assess the effect of ventilatory parameters at ICU admission.

Both models confirm that age is one of the strongest predictors of ICU survival, with a probability of death reaching almost $100 \%$ in older patients. The strong association between age and mortality is a constant finding in COVID-19 literature $(4,14)$. Male gender is a negative predicting factor, a finding confirmed by previous studies (15). An etiological justification might be linked to a difference between the sexes in cellular immunity as males present a poorer T-cell activation and an increase in proinflammatory cytokines (16).

Chronic kidney disease (CKD) is highly correlated with mortality in our data, and this may be related to several factors. CKD affects older patients(17) that, as already demonstrated widely (3) and confirmed by our models, are particularly fragile when hospitalized for COVID-19. Secondly, all stages of CKD are associated with an increased risk of premature mortality from all causes(18) and thirdly, CKD is associated in up to two-thirds of the cases with diabetes and hypertension (19), a proxy for older, multimorbid patients. (4) COVID-19 disease is also associated with new onset acute kidney injury, that may further worsen previous kidney disfunction, leading to organ failure. $(20,21)$

We found a strong association between chronic obstructive pulmonary disease (COPD) and mortality. COPD patients have both an increased risk of COVID-19 disease, and a poorer prognosis, with higher rates of hospitalization and mortality. (22) COPD is an independent predictor of mortality in patients admitted to ICU for COVID-19 pneumonia. (4)

Diabetes Mellitus is associated with mortality in our results. Type-2 diabetes mellitus is more frequent in older patients, male gender, and is part of the metabolic syndrome with hypertension and obesity, which was previously demonstrated to have strong association with COVID-19 outcomes.(23) The association of diabetes and survival has been questioned by other studies, where the association was lost after controlling for other factors $(4,24)$.

Chronic therapy with ACE inhibitors and ARBs were associated with higher mortality in this analysis. Initial reports linked the possible pharmacodynamics of these classes of drugs to an up-regulation of ACE2 expression(25) and a consequent increase in the availability of target molecules for SARS-CoV2(26). This association has been proven wrong by Mancia et al(27), who performed a large populationbased case-control study demonstrating that use of ACE inhibitors and ARBs was more frequent among 
COVID-19 patients due to their higher prevalence of cardiovascular disease, without evidence linking those drugs to an higher risk of infection by SARS-CoV-2.

Compared to Model A, Model B overly relies on the type of ventilatory assistance at ICU admission (mechanical ventilation vs Non-invasive ventilation vs spontaneous breathing), at the expense of an higher variance compared to model A. Among the other ventilatory variables in the first $24 \mathrm{~h}$, only the fraction of inspiratory oxygen $\left(\mathrm{FiO}_{2}\right)$ administered during the first hours was demonstrated to be inversely associated with mortality in the final $\mathrm{B}$ model.

\section{Limitations}

The study presents several limitations. First, it is an observational study based on operative data daily collected during an emergency crisis by a regional coordination center, hindering the quality of data assured by a research targeted database. Despite being a limitation, it was one of the main goals of the models: to test the ability of machine learning models during the escalation phase of the spread of SARS$\mathrm{CoV} 2$ where an hold-out validation cannot be retrieved. Some variables that could be useful to increase the predictive performance of the model could not be collected, including data about comorbidities (i.e. CKD Stage, hypertension severity) and other physiological parameters (weight, body mass index, more complex ventilatory data, patient frailty). Availability of more data could probably reduce class imbalance in this population.

The number of patients and data included in this study is not comparable with big data analysis, where machine learning techniques outperform classic statistical. However, with this study we were able to demonstrate that machine learning approach may be used even with smaller dataset in an emergency setting and reach high predictive performance.

\section{Conclusions}

Supervised machine learning models demonstrated good performance in the prediction of 28-day mortality. Lower performance was demonstrated at 7 and 14 days. As the model improves with further data, it may be used as a score of severity before ICU admission to stratify patients according to survival probability. Machine learning techniques may be useful in emergency phases, to reach higher predictive performance with reduced human supervision using complex data.

\section{Declarations}

\section{Ethics approval and consent to participate:}

The institutional ethics board of Fondazione IRCCS Ca' Granda Ospedale Maggiore Policlinico, Milan, approved this study and waived the need for informed consent from individual patients owing to the retrospective nature of the study. This study followed the Strengthening the Reporting of Observational Studies in Epidemiology (STROBE) reporting guideline. 
Consent for publication: not applicable.

Availability of data and materials: the dataset generated and analyzed during the current study are not publicly available at the moment as we are still contacting and waiting for the authorization of every center involved in the formation of the dataset.

Competing interests: the authors declare that they have no competing interests.

Funding: not applicable.

\section{Authors' contributions:}

On behalf of the Lombardy ICU Network:

Drs Greco and Angelotti contributed equally as co-first authors.

Concept and design: MG, GA

Acquisition, analysis, or interpretation of data: MG, GA, PFC, AZ

Drafting of the manuscript: MG, GA, PFC, NS

Critical revision of the manuscript for important intellectual content: AZ, EC, Alessandro Protti, Antonio Pesenti, GG, MC

Supervision: Antonio Pesenti, GG, MC

Acknowledgements: The list of all the collaborators of the Lombardy ICU network involved in this study will be provided as soon as possible as we finalize the approval from every center involved.

\section{References}

1. Chen N, Zhou M, Dong X, Qu J, Gong F, Han Y, et al. Epidemiological and clinical characteristics of 99 cases of 2019 novel coronavirus pneumonia in Wuhan, China: a descriptive study. Lancet. 2020.

2. WHO announces COVID-19 outbreak a pandemic. 2020 Mar 12 [cited 2020 Jun 25]; Available from: https://www.euro.who.int/en/health-topics/health-emergencies/coronavirus-covid19/news/news/2020/3/who-announces-covid-19-outbreak-a-pandemic.

3. Grasselli G, Zangrillo A, Zanella A, Antonelli M, Cabrini L, Castelli A, et al. Baseline Characteristics and Outcomes of 1591 Patients Infected With SARS-CoV-2 Admitted to ICUs of the Lombardy Region, Italy. JAMA. 2020.

4. Grasselli G, Greco M, Zanella A, Albano G, Antonelli M, Bellani G, et al. Risk Factors Associated with Mortality among Patients with COVID-19 in Intensive Care Units in Lombardy, Italy. JAMA Intern Med [Internet]. 2020 [cited 2020 Sep 16]; Available from: https://pubmed.ncbi.nlm.nih.gov/32667669/. 
5. Karagiannidis C, Mostert C, Hentschker C, Voshaar T, Malzahn J, Schillinger G, et al. Case characteristics, resource use, and outcomes of 10021 patients with COVID-19 admitted to 920 German hospitals: an observational study. Lancet Respir Med [Internet]. 2020 Sep 1 [cited 2020 Sep 17];8(9):853-62. Available from: www.thelancet.com/respiratoryVol.

6. Gupta S, Hayek SS, Wang W, Chan L, Mathews KS, Melamed ML, et al. Factors Associated with Death in Critically III Patients with Coronavirus Disease 2019 in the US. JAMA Intern Med [Internet]. 2020 [cited 2020 Sep 17]; Available from: https://jamanetwork.com/.

7. Arentz M, Yim E, Klaff L, Lokhandwala S, Riedo FX, Chong M, et al. Characteristics and Outcomes of 21 Critically III Patients With COVID-19 in Washington State. JAMA [Internet]. 2020/03/20. 2020; Available from: https://jamanetwork.com/journals/jama/articlepdf/2763485/jama_arentz_2020_ld_200020.pdf. 8. Greco M, Caruso PF, Cecconi M. Artificial Intelligence in the Intensive Care Unit. Semin Respir Crit Care Med [Internet]. 2020 [cited 2021 Jan 13]; Available from: https://pubmed.ncbi.nlm.nih.gov/33152770/.

9. Deasy J, Liò P, Ercole A. Dynamic survival prediction in intensive care units from heterogeneous time series without the need for variable selection or curation. Sci Rep [Internet]. 2020 Dec 1 [cited 2021 Jan 13];10(1). Available from: https://pubmed.ncbi.nlm.nih.gov/33335183/.

10. Grasselli G, Pesenti A, Cecconi M. Critical Care Utilization for the COVID-19 Outbreak in Lombardy, Italy: Early Experience and Forecast During an Emergency Response. JAMA [Internet]. 2020/03/14. 2020 [cited 2020 May 3]; Available from: https://jamanetwork.com/journals/jama/articlepdf/2763188/jama_grasselli_2020_vp_200049.pdf.

11. Tang Y, Zhang YQ, Chawla NV. SVMs modeling for highly imbalanced classification. IEEE Trans Syst Man Cybern Part B Cybern. 2009;39(1):281-8.

12. He H, Bai Y, Garcia EA, Li SADASYN. Adaptive Synthetic Sampling Approach for Imbalanced Learning [Internet]. ieeexplore.ieee.org. [cited 2021 Jan 15]. Available from: https://ieeexplore.ieee.org/abstract/document/4633969/.

13. Pears R, Finlay J, Connor AM. Synthetic minority over-sampling technique (SMOTE) for predicting software build outcomes. In: Proceedings of the International Conference on Software Engineering and Knowledge Engineering, SEKE. Knowledge Systems Institute Graduate School; 2014. p. 546-51.

14. O'Brien J, Du KY, Peng C. Incidence, clinical features, and outcomes of COVID-19 in Canada: impact of sex and age. J Ovarian Res [Internet]. 2020 Dec 1 [cited 2021 Jan 14];13(1). Available from: https://pubmed.ncbi.nlm.nih.gov/33234144/.

15. Pastor-Barriuso R, Pérez-Gómez B, Hernán MA, Pérez-Olmeda M, Yotti R, Oteo J, et al. SARS-CoV-2 infection fatality risk in a nationwide seroepidemiological study. medRxiv. 2020;1-13.

16. Takahashi T, Wong P, Ellingson M, Lucas $C$, Klein J, Israelow B, et al. Sex differences in immune responses to SARS-CoV-2 that underlie disease outcomes. medRxiv Prepr Serv Heal Sci. 2020.

17. Bill F, Foundation MG. Global, regional, and national burden of chronic kidney disease, 1990-2017: a systematic analysis for the Global Burden of Disease Study 2017. 2017. 
18. Hill NR, Fatoba ST, Oke JL, Hirst JA, Callaghan AO, Lasserson DS, et al. Global Prevalence of Chronic Kidney Disease - A Systematic Review and Meta-Analysis. 2016;1-18.

19. KDIGO. Official Journal of the international supplements Society of nephrology KDIGO 2012 Clinical Practice Guideline for the Evaluation and Management of Chronic Kidney Disease. Kidney Int Suppl. 2013.

20. Louis G, Belveyre T, Goetz C, Gaci R, Dinot V. Acute kidney injury in severe SARS-CoV-2 infection: An experience report in Eastern France [Internet]. Vol. 40, Anaesthesia Critical Care and Pain Medicine. Elsevier Masson s.r.l.; 2021 [cited 2021 Jan 14]. p. 100788. Available from: https://www.ncbi.nlm.nih.gov/pmc/articles/PMC7700728/.

21. Gabarre P, Dumas G, Dupont T, Darmon M, Azoulay E, Zafrani L. Acute kidney injury in critically ill patients with COVID-19 [Internet]. Vol. 46, Intensive Care Medicine. Springer; 2020 [cited 2021 Jan 14]. p. 1339-48. Available from: https://pubmed.ncbi.nlm.nih.gov/32533197/.

22. Graziani D, Soriano JB, Del Rio-Bermudez C, Morena D, Díaz T, Castillo M, et al. Characteristics and Prognosis of COVID-19 in Patients with COPD. J Clin Med [Internet]. 2020 Oct 12 [cited 2021 Jan 14];9(10):3259. Available from: https://pubmed.ncbi.nlm.nih.gov/33053774/.

23. Simonnet A, Chetboun M, Poissy J, Raverdy V, Noulette J, Duhamel A, et al. High Prevalence of Obesity in Severe Acute Respiratory Syndrome Coronavirus-2 (SARS-CoV-2) Requiring Invasive Mechanical Ventilation. Obesity [Internet]. 2020 Jul 1 [cited 2021 Jan 14];28(7):1195-9. Available from: https://pubmed.ncbi.nlm.nih.gov/32271993/.

24. Izzi-Engbeaya C, Distaso W, Amin A, Yang W, Idowu O, Kenkre JS, et al. Adverse outcomes in COVID19 and diabetes: a retrospective cohort study from three London teaching hospitals. BMJ Open Diabetes Res Care [Internet]. 2021 Jan 6 [cited 2021 Jan 14];9(1):e001858. Available from: https://drc.bmj.com/lookup/doi/10.1136/bmjdrc-2020-001858.

25. Soler MJ, Barrios C, Oliva R, Batlle D. Pharmacologic modulation of ACE2 expression. Curr Hypertens Rep. 2008;10(5):410-4.

26. Esler M, Esler D. Can angiotensin receptor-blocking drugs perhaps be harmful in the COVID-19 pandemic? J Hypertens. 2020;38(5):781-2.

27. Mancia G, Rea F, Ludergnani M, Apolone G, Corrao G. Renin-angiotensin-aldosterone system blockers and the risk of COVID-19. N Engl J Med. 2020;382(25):2431-40.

\section{Figures}




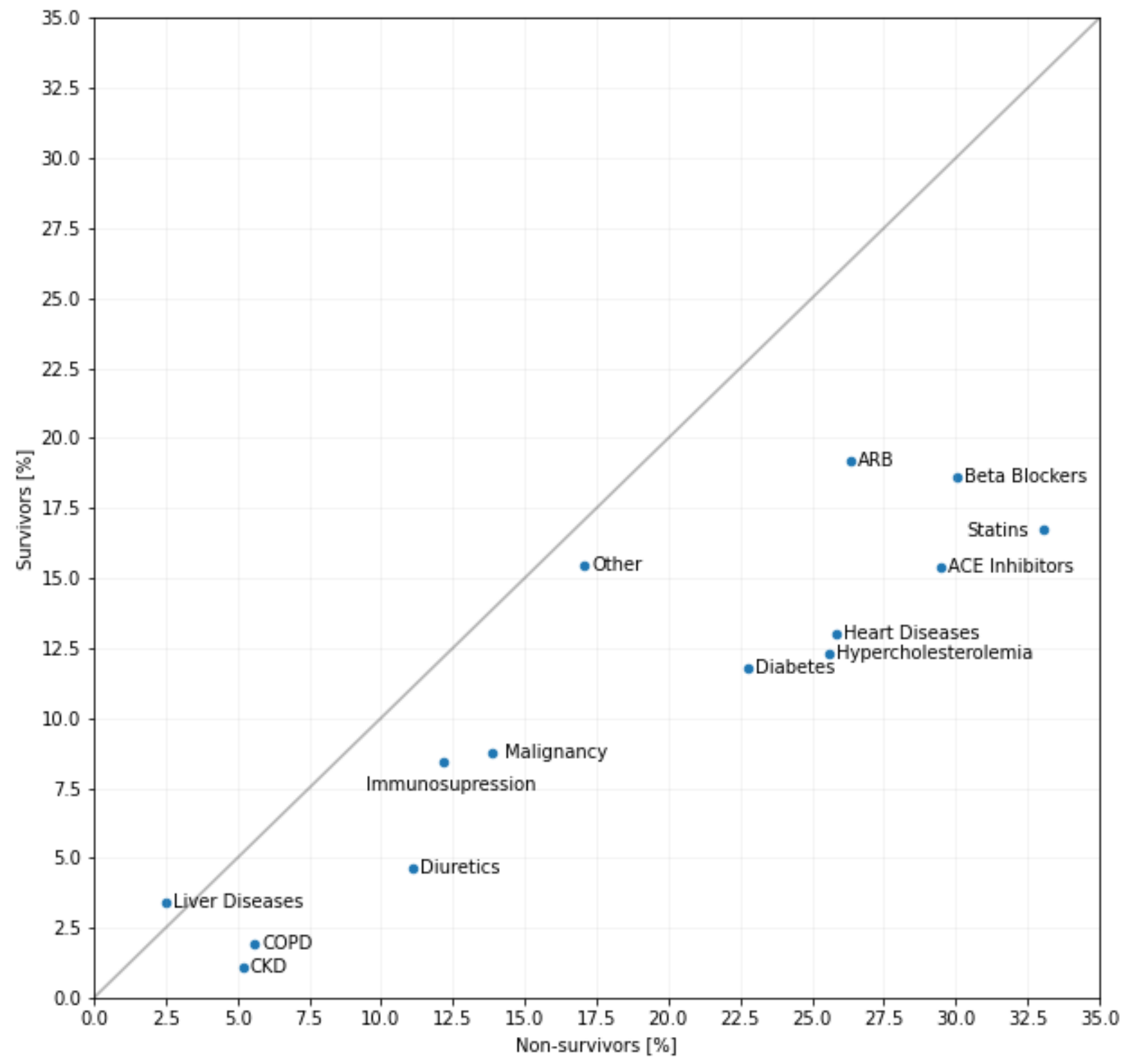

Figure 1

Scatter plot summarizing the proportion of comorbidities in Survivors and Non-Survivors. 


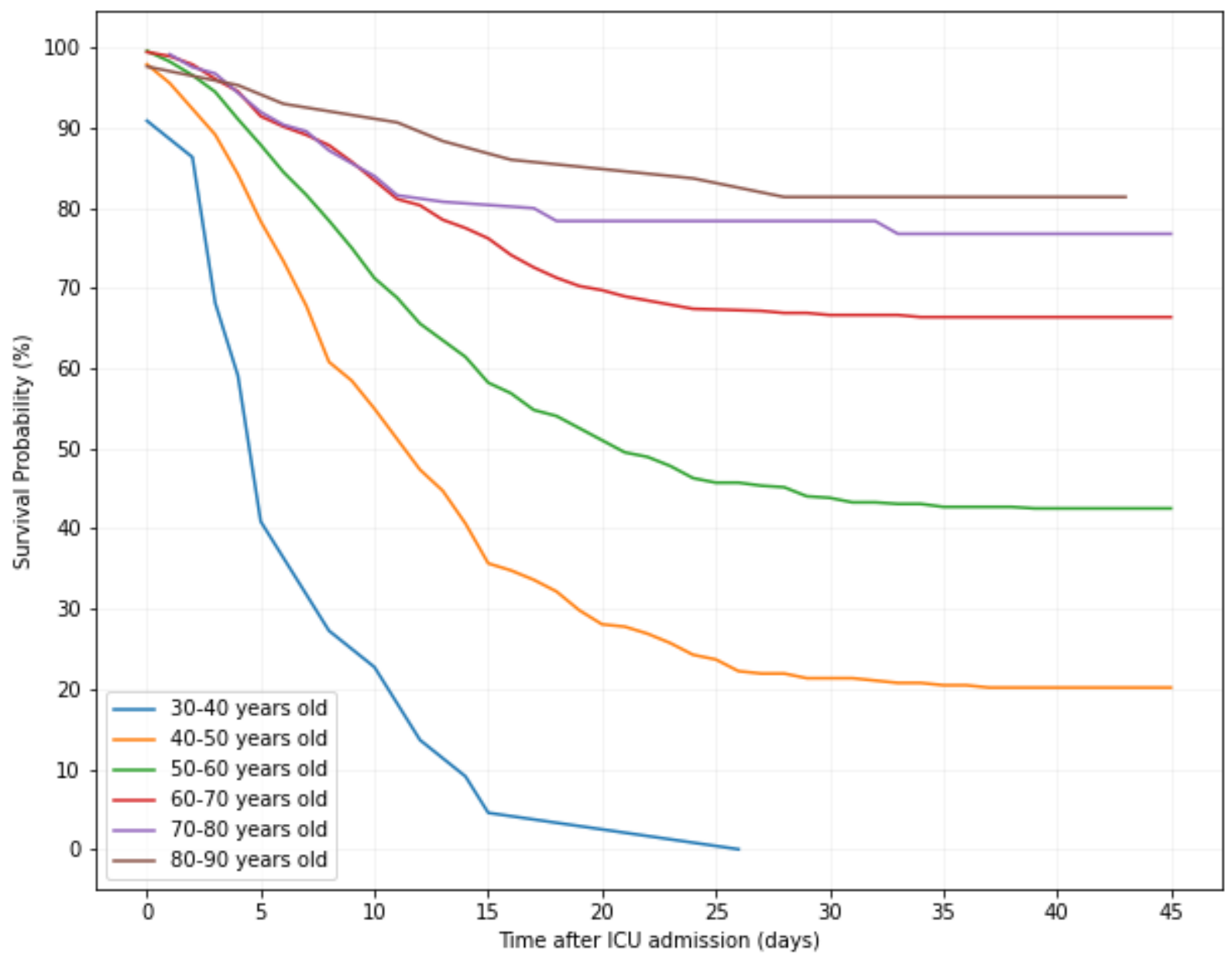

Figure 2

Kaplan-Maier curve by age 

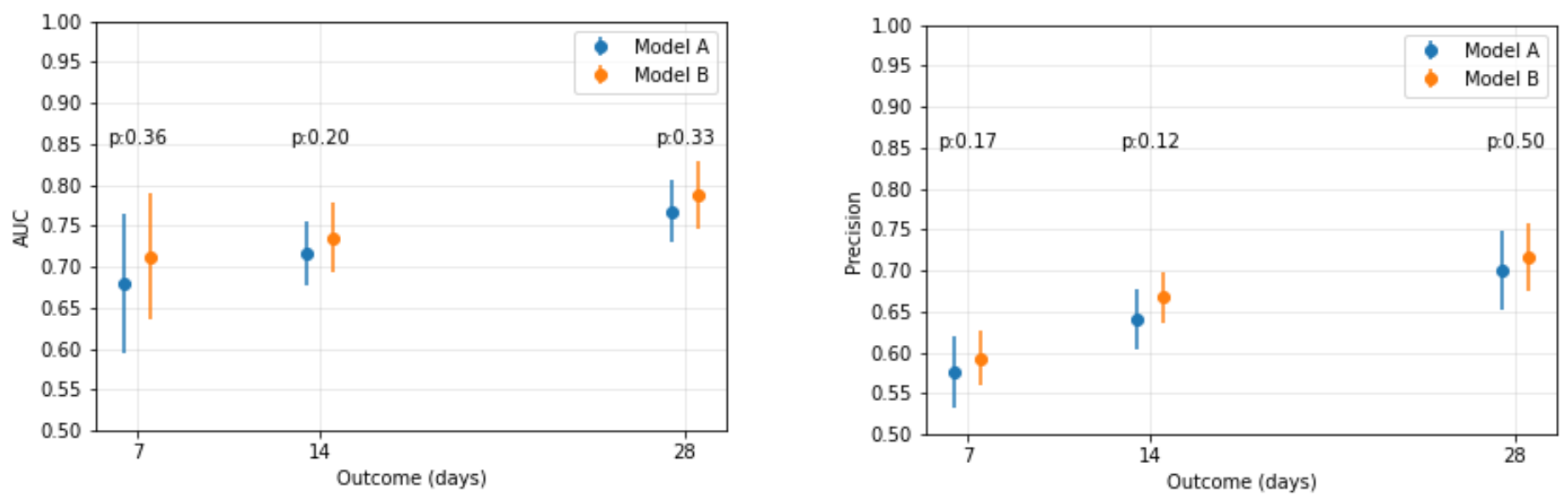

\section{A}

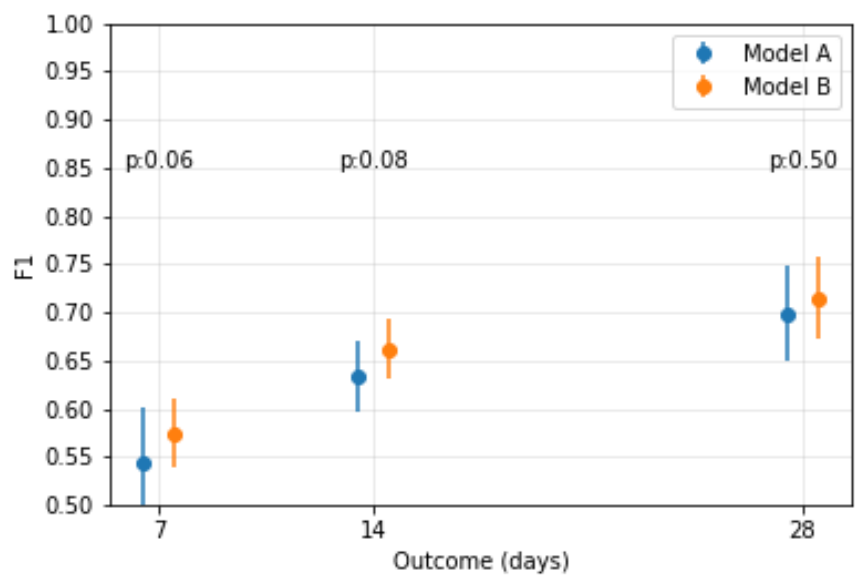

B
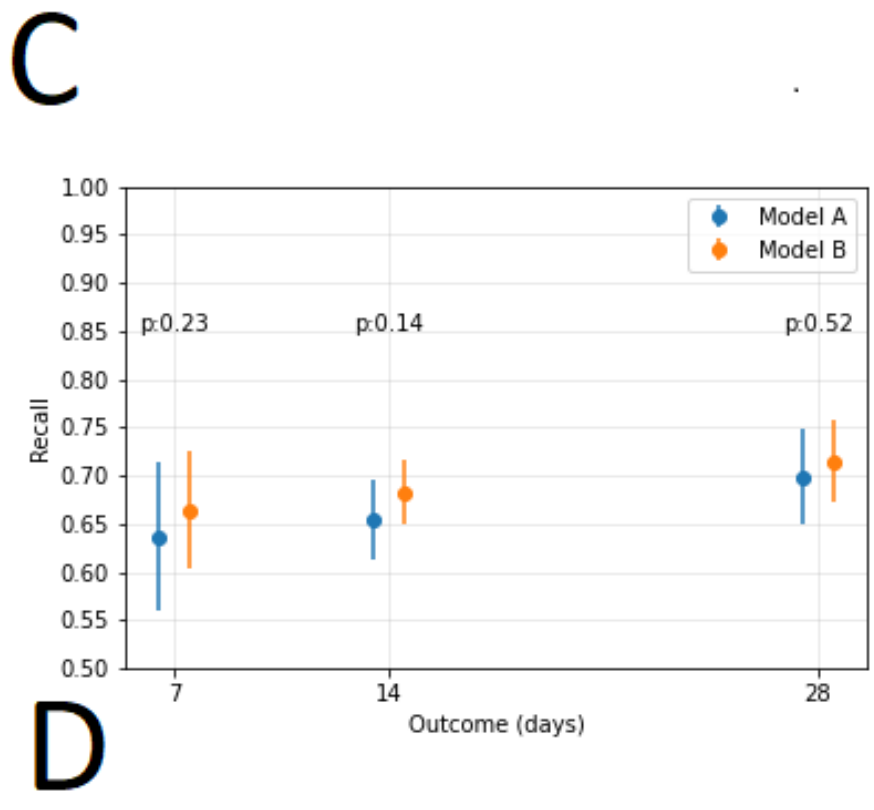

Figure 3

Performance model A and model $B$ at different time points. The dot represents the mean score and line the standard deviation across folds. 3A) Area Under the Curve; 3B) F1 score; 3C) Precision; 3D) Recall

\section{Supplementary Files}

This is a list of supplementary files associated with this preprint. Click to download.

- supplementalmaterialv6.docx 